\title{
Aflibercept in persistent neovascular AMD: comparison of different treatment strategies in switching therapy
}

F Ricci ${ }^{1,3}$, M Parravano ${ }^{2,3}, \mathrm{~F}^{2}$ Regine ${ }^{1}$, M Sciamanna ${ }^{2}$, M Tedeschi $^{2}$, F Missiroli ${ }^{1}$ and M Varano ${ }^{2}$

\begin{abstract}
Purpose To evaluate the effects of aflibercept administered according to a pro re nata (PRN) or Fixed Regimen to patients with neovascular AMD and persistent intraretinal/ subretinal fluid (IRF/SRF) despite three consecutive ranibizumab injections. Methods Patients were switched to aflibercept injection (IVA) administered according to a PRN or to a fixed regimen for 1 year in two different retina centers. At baseline each patient underwent a complete ophthalmologic evaluation, including bestcorrected visual acuity assessment (BCVA ETDRS chart), fluorescein, and indocyanine green angiography and OCT.

Results Each group included 36 eyes. After
\end{abstract} 1 year the PRN group showed BCVA stabilization (63 vs 60 letters, $P=0.33$ ), whereas fixed regimen group showed significant BCVA improvement (68 vs $71, P=0.008$ ). The median central retinal thickness decreased by $94 \mu \mathrm{m}$ in the PRN $(P=0.002)$ and by $148 \mu \mathrm{m}$ in the fixed regimen group $(P \leq 0.001)$. Complete IRF/SRF reabsorption was found in $58 \%$ of eyes in the PRN and in $42 \%$ of eyes in the fixed regimen group. At 1-year visit, the percentage of eyes with pigment epithelium detachment did not significantly decrease, but a height reduction was recorded in both groups. The median number of IVA was 3.5 in the PRN and 7 in the fixed regimen group.

Conclusion The switch to aflibercept with both treatment strategies enabled improvement in morphological parameters and stabilization of visual acuity. BCVA improvement and reduction in vision loss with reduction in retinal thickness, fluid and PED height was achieved with the fixed regimen in previously treated nAMD after 1 year.

Eye (2016) 30, 1077-1083; doi:10.1038/eye.2016.95; published online 27 May 2016

\section{Introduction}

Age-related macular degeneration (AMD) is a leading cause of adult blindness in the developed world. ${ }^{1}$ Severe visual loss from AMD is caused by subfoveal choroidal neovascularization, which is the hallmark of neovascular AMD (nAMD).

The use of intravitreal anti-vascular endothelial growth factor (VEGF) therapy currently is the standard of care for nAMD. The most commonly used agents, bevacizumab and ranibizumab, reduce exudative fluid and have been shown to improve best-corrected visual acuity (BCVA) in eyes with nAMD compared with controls. ${ }^{2-4}$

Aflibercept is a new anti-VEGF agent, which is a recombinant fusion protein consisting of key human VEGF receptor 1 and 2 extracellular domains (VEGFR1 and VEGFR2) fused to the Fc domain of human IgG1. ${ }^{5,6}$ This protein binds VEGF-A, VEGF-B, and placental growth factor (PIGF), thus inhibiting the binding and the activation of VEGF receptors. 5,6

Recently, the results of a randomized clinical trial comparing aflibercept (IAI $2 \mathrm{mg}$, dosed every 2 months after 3 initial monthly loading injections) vs ranibizumab ( $0.5 \mathrm{mg}$ every month) in naive nAMD (the VIEW trial) revealed that visual outcomes were similar with the two agents at 52 weeks and also after 96 weeks of follow up. ${ }^{7,8}$

The availability of different anti-VEGF therapies offers the opportunity to switch from one anti-VEGF agent to another in subjects who still have active lesions after anti-VEGF treatment. ${ }^{9-20}$ There have been several case series describing the outcome of switching from ranibizumab and/or bevacizumab to aflibercept in poor responders with nAMD. Different treatment regimens of aflibercept have been
${ }^{1}$ Ophthalmology Department, University of Rome Tor Vergata, Rome, Italy

${ }^{2}$ Ophthalmology Department, Fondazione G.B. Bietti-IRCCS, Rome, Italy

Correspondence: M Parravano, Ophthalmology Department, Fondazione G.B. Bietti-IRCCS, Via Livenza n 3, 00198 Rome, Italy

Tel:+390685356727;

Fax:+390684242333

E-mail: criparra@tin.it

${ }^{3}$ These authors contributed equally to this work.

Received: 15 November 2015

Accepted in revised form: 25 February 2016 Published online: 27 May 2016 
adopted for the switch and no clear dosing recommendations have been made.

The purpose of this study was to evaluate the long-term effects of two different treatment strategies of intravitreal aflibercept (IVA; pro re nata (PRN) or fixed) in patients with nAMD and persistent retinal fluid after at least three monthly intravitreal ranibizumab injections.

\section{Materials and methods}

In this retrospective clinical study, patients affected by nAMD, showing persistent activity on spectral-domain optical coherence tomography (SD-OCT) and treated with intravitreal ranibizumab (IVR) were switched to IVA injection administered according to a PRN or to a fixed regimen at the Department of Ophthalmology of IRCCS Fondazione G.B. Bietti and University of Rome Tor Vergata. The study protocol adhered to the tenets of the Declaration of Helsinki Principles, was approved by the local Institutional Review Board. Each patient signed an informed consent prior to the intravitreal injections.

Eligibility criteria were: nAMD with persistent intraretinal/subretinal fluid (IRF/SRF) after at least three monthly consecutive IVR injections as evaluated by means of SD-OCT.

The key exclusion criteria included: history of vitrectomy, trabulectomy, surgery for retinal detachment, or any intraocular or periocular surgery in the study eye (within 3 months of day 1), uncontrolled glaucoma, or uveitis or any other ocular disease able to confound the assessment of safety and/or efficacy of treatment. Moreover, patients with uncontrolled systemic hypertension, peripheral vascular disease and history of thromboembolism or stroke were excluded.

Each eye received IVA at the recommended dose of $2 \mathrm{mg}(0.05 \mathrm{ml})$ every 4 weeks for 12 weeks (loading phase), followed by intravitreal injections 2 months apart in the Fixed Regimen group or a single injection followed by a PRN regimen based on predefined re-treatment criteria. Following the first IVA, each patient in the PRN group was examined monthly (4 weeks) over a 12-month follow-up period. Additional re-treatment was administered if IRF/SRF was confirmed on SD-OCT or new hemorrhages were detected upon fundus examination or choroidal neovascularization (CNV) leakage was detected at fluorangiography.

BCVA measurement with ETDRS charts (letter score), slit lamp biomicroscopy, intraocular pressure assessment by Goldman applanation tonometry and SD-OCT Spectralis (version 1.5.12.0; Heidelberg Engineering, Heidelberg, Germany) were performed at a scheduled monthly visit in the PRN group and before each scheduled injection. The Volume Fast program (version
1.5.12.0; Heidelberg Engineering) was used for SD-OCT scanning and central retinal thickness (CRT), which corresponds to the mean thickness in the central $1 \mathrm{~mm}$ diameter area, was evaluated. Fluorescein angiography and Indocyanine green angiography (FA and ICGA, HRA2 Heidelberg Engineering, and TRC-50X; Topcon Instrument Corp., Tokyo, Japan) were performed at baseline and at the discretion of the investigator during the study.

Intravitreal injections of aflibercept were administered according to the national and international guidelines for intravitreal injections. ${ }^{21}$

The study's primary outcome was the assessment of the mean BCVA (ETDRS letter score) at 12 months in both treatment regimen groups.

Secondary outcomes included the mean change in BCVA, the proportion of patients losing 5-15 ETDRS letters, the change in CRT, as well as the changes in IRF/ $\mathrm{SRF}$, the presence of pigment epithelium detachment (PED), the subfoveal height of PED (manual measurement by SD-OCT caliper), and the number of injections.

The Wilcoxon test and the Mann-Whitney test (paired and unpaired depending upon the groups) were carried out to evaluate the changes in BCVA, CRT, and PED height, while the Fisher exact test was performed for the comparison of proportions. The level of significance was set at $P<0.05$.

\section{Results}

The analysis identified 72 eyes of 72 patients fully satisfying inclusion and exclusion criteria. During the study period 36 eyes received aflibercept according to the PRN regimen (PRN group) and 36 eyes received the loading dose plus a fixed bimonthly injection (fixed regimen group). Demographics and clinical characteristics of all patients are summarized in Table 1.

In the PRN group 13 out of 26 cases of occult CNV (50\%) had serous PED. ICG angiography of these lesions disclosed four cases of retinal angiomatous proliferation (RAP) and one case of polypoidal choroidal vasculopathy (PCV).

In the fixed regimen group, 22 cases have occult CNV $(61.1 \%)$ at FA. Twenty out of 22 cases of occult CNV $(90.9 \%)$ had serous PED. ICG angiography of these lesions revealed one case of RAP and two of PCV.

At the baseline examination, the two groups did not differ with regard to age, gender distribution, previous number of ranibizumab injections, the time between the CNV diagnosis and the first aflibercept injection, CNV type, and proportion with PED.

At baseline, the median BCVA value was 63 letters in the PRN group. At 4-, 8-, and 12-month examinations, the BCVA was stable and the final median value was 60 
Table 1 Baseline demographic and clinical characteristics of the patients

\begin{tabular}{|c|c|c|c|}
\hline Baseline characteristics & PRN group & $\begin{array}{l}\text { Fixed regimen group } \\
\text { (loading phase }+2 q 8 \text { ) }\end{array}$ & P-value \\
\hline Number of patients & $36(50 \%)$ & $36(50 \%)$ & 1.0 \\
\hline Median age (range) & $79(59-93)$ & $77(52-85)$ & 0.17 \\
\hline Gender & 19 F (53\%)-17 M (47\%) & 14 F (39\%)/22 M (61\%) & 0.34 \\
\hline $\begin{array}{l}\text { Median number of previous ranibizumab injections } \\
(n, 95 \% \mathrm{CI})\end{array}$ & $5(4.5-7.5)$ & $6.5(4-9.5)$ & 0.78 \\
\hline Median time of CNV diagnosis (months, $95 \% \mathrm{CI}$ ) & $18(14-24)$ & $22(11-31)$ & 0.71 \\
\hline \multirow[t]{2}{*}{ CNV type } & C: 10 (28\%), O: 26 (72\%) & C: $14(39 \%)$, O: $22(61 \%)$ & 0.21 \\
\hline & PED: $13(36 \%)$ & PED $20(56 \%)$ & 0.15 \\
\hline
\end{tabular}

Abbreviations: C, classic; CNV, choroidal neovascularization; fixed regimen, loading phase+bimonthly injections; $\mathrm{O}$, occult; PED, pigment epithelium detachment; PRN, pro re nata strategy.

Table 2 Median best-corrected visual acuity (ETDRS letter) during the 1-year follow-up study

\begin{tabular}{|c|c|c|c|c|}
\hline & Baseline & 4 Months & 8 Months & 12 Months \\
\hline All patients, median ETDRS letter (95\% CI) & $65(60-70)$ & 65 (61-72, p: 0.08) & 66 (60-72, p: 0.87) & 67 (60-70, p: 0.24) \\
\hline PRN regimen & $63(52-71)$ & $60(55-68, \mathrm{p}: 0.54)^{\mathrm{a}}$ & $\begin{array}{c}60 \text { (52-68, p: } 0.54)^{\mathrm{a}} \\
\text { vs baseline } 0.92^{\mathrm{b}}\end{array}$ & $\begin{array}{c}60(54-64, \mathrm{p}: 0.91)^{\mathrm{a}} \\
\text { vs baseline } 0.33^{\mathrm{b}}\end{array}$ \\
\hline Fixed regimen group (Loading phase $+2 \mathrm{q} 8$ ) & $68(60-72)$ & $71(64-77, \mathrm{p}: 0.02)^{\mathrm{a}}$ & $\begin{array}{l}74(63-78, p: 0.75)^{\mathrm{a}} \\
\text { vs baseline } 0.0 .006^{\mathrm{b}}\end{array}$ & $\begin{array}{l}71(65-79, \text { p: } 0.73)^{\mathrm{a}} \\
\text { vs baseline } 0.008^{\mathrm{b}}\end{array}$ \\
\hline PRN group vs fixed regimen group & (p: 0.31) & (p: 0.03) & (p: 0.02) & (p: 0.01$)$ \\
\hline
\end{tabular}

Abbreviations: BCVA, best-corrected visual acuity; fixed regimen, loading phase+bimonthly injections; PRN, pro re nata strategy.

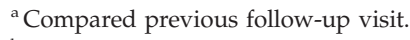

${ }^{\mathrm{b}}$ Compared with baseline value.

The median BCVA showed stabilization in the PRN group over the whole study period; a statistically significant improvement in BCVA was recorded at 4-month examination in the fixed regimen group, which was preserved on subsequent visits at 8 and 12 months.

Table 3 Central retinal thickness $(\mu \mathrm{m})$ during the 1-year follow-up study

\begin{tabular}{|c|c|c|c|c|}
\hline & Baseline & 4 Months & 8 Months & 12 Months \\
\hline All patients, median CMT $(95 \% \mathrm{CI})$ & $403(384-476)$ & $297(268-321, \mathrm{p}:<0.0001)^{\mathrm{a}}$ & $310(272-337, \mathrm{p}: 0.74)^{\mathrm{a}}$ & 266 (253-28, p: 0.81) $)^{\mathrm{a}}$ \\
\hline PRN regimen & $346(315-414)$ & $256(249-284, \text { p: } 0.02)^{a}$ & $\begin{array}{c}260(250-309, \text { p: } 0.83)^{\mathrm{a}} \\
\text { vs baseline } 0.045\end{array}$ & $\begin{array}{c}252(239-265, \text { p: } 0.158)^{\mathrm{a}} \\
\text { vs baseline } 0.002\end{array}$ \\
\hline Fixed regimen group (loading phase+2q8) & $480(397-539)$ & $321(297-380, \mathrm{p}:<0.0001)^{\mathrm{a}}$ & $\begin{array}{c}333(308-386, \text { p: } 0.49)^{\mathrm{a}} \\
\text { vs baseline }<0.001\end{array}$ & $\begin{array}{c}332(310-375, \text { p: } 0.56)^{\mathrm{a}} \\
\text { vs baseline }<0.001\end{array}$ \\
\hline PRN group $v s$ fixed regimen group & $(\mathrm{p}:<0.0001)$ & (p: 0.01) & (p: 0.02) & $(P<0.001)$ \\
\hline
\end{tabular}

Abbreviations: fixed regimen, loading phase+bimonthly injections; PRN, pro re nata strategy.

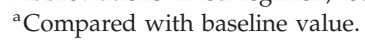

Statistically significant reduction in median CMT was recorded in both subgroups at the 4-month visit. Afterwards, stabilization in median CMT values was maintained until the end of the 1-year follow-up study in both groups.

letters ( $P=0.33$ vs baseline; Table 2). The fixed regimen group showed a statistically significant improvement from the baseline value (from 68 letters to 71 letters) at 4-month examination $(P=0.02)$. After this examination BCVA stabilized and at month 12 it still was 71 letters ( $P=0.008$ vs baseline).

In the PRN group $25 \%$ of patients (9/36) lost $5-15$ letters at 12 months; only 1 patient lost $>15$ letters. In the fixed regimen group $8 \%$ of patients (3/36) lost $5-15$ letters at 12 months; no patient lost $>15$ letters. At 12 months, mean change in BCVA was significantly better in the fixed regimen group (median, 2.5 letters; IC 95\% - 0.99/ - 5) than in the PRN group (median, -0.5 letters; IC 95\% $-4.50 /-2.00)$.

A significant reduction in the median CRT was recorded in both groups at the 4-month visit. Subsequently, median CRT values stabilized until the end of the study. The CRT reduction vs baseline was still significant at 12 months in both groups (PRN $P=0.002$, fixed $P<0.001)$. The CRT mean change by month 12 was 
similar $(P=0.20)$ in the PRN (median -66.5; IC 95\% $-111 /-20)$ and Fixed Regimen group (median - 81; IC 95\% - 146/ - 40; Table 3).

At month 12 in both groups, IRF/SRF disappeared in $50 \%$ of eyes; the proportion of dry patients was $58 \%$ in the PRN group $(21 / 36 ; P<0.0001)$ and $42 \%(15 / 36)$ in the fixed regimen group $(P<0.001$; Table 4$)$.

A total of 33 eyes had PED at the baseline examination, 13 in the PRN group and 20 in the fixed regimen group. After 1 year, complete PED flattening was recorded in four patients, three in the PRN and one in the fixed regimen group (Table $5 \mathrm{a}$ ).

However, a greater reduction in the median subfoveal PED height was achieved at 12-month visit with the Fixed regimen (186 vs 90 micron, 52\% reduction) than with PRN regimen (262 vs 174 micron, 31.9\% reduction; Table 5b).

The mean number of aflibercept injections administered by month 12 after the switch was $3.5 \pm 1.6( \pm S D$, median:
4; min 1; max 7) in the PRN group and 7 (median: 7) in the fixed regimen group. The average injection free interval in PRN group was $3.48 \pm 1.42$ months and in the fixed group was 1.71 months $(P<0.0001)$.

The highest number of injections in the PRN regimen was administered in the first 3 months $(12 / 36,33 \%)$ and the lowest at month $10(1 / 36,2.8 \%)$.

The total number of intravitreal injections in the whole follow-up period was 127 in the PRN and 252 in the fixed regimen group.

Concerning the number of visits in the PRN group (including the end study visit) patients attended a total of 396 out of 468 planned visits (15.4\% visit loss), with a mean number of visits of $11.02 \pm 1.86$ (7-13).

In the fixed regimen group, despite the high number of injections, they attended only 144 visits, equivalent to just over one third $(36 \%)$ of the visits attended in the PRN group, with a mean number of visits of 4 .

Table 4 Distribution of the proportion of patients with intraretinal/subretinal fluid on optical coherence tomography examination at baseline and 12-month follow up

\begin{tabular}{lccc}
\hline & Baseline & 12 Months & P-value \\
\hline All patients (presence/72) & $100 \%(72 / 72)$ & $50 \%(36 / 72)$ & $<0.0001$ \\
PRN regimen & $100 \%(36 / 36)$ & $42 \%(15 / 36)$ & $<0.0001$ \\
Fixed regimen group (Loading phase+2q8) & $100 \%(36 / 36)$ & $58 \%(21 / 36)$ & $<0.0001$ \\
PRN group vs fixed regimen group & (p: 1.00$)$ & $(\mathrm{p}: 0.23)$ & \\
\hline
\end{tabular}

Abbreviations: fixed regimen, loading phase+bimonthly injections; PRN, pro re nata strategy.

The intraretinal/subretinal fluid displayed a statistically significant decrease during the period study in both groups.

Table 5a Distribution of the proportion of patients with pigment epithelial detachment on optical coherence tomography examination at baseline and 12-month follow-up

\begin{tabular}{lccc}
\hline & Baseline & 12 Months & P-value \\
\hline All patients (presence/72) & $46 \%(33 / 72)$ & $40 \%(29 / 72)$ & 0.40 \\
PRN regimen & $36 \%(13 / 36)$ & $28 \%(10 / 36)$ & 0.46 \\
Fixed regimen group (loading phase+2q8) & $56 \%(20 / 36)$ & $53 \%(19 / 36)$ & 0.99 \\
PRN group vs fixed regimen group & (p: 0.15$)$ & (p: 0.05$)$ & \\
\hline
\end{tabular}

Abbreviations: fixed regimen, loading phase+bimonthly injections; PED, pigment epithelial detachment; PRN, pro re nata strategy.

No remarkable reduction in the presence of PED was recorded in both groups during the period study.

Table $5 \mathbf{b}$ Variation in pigment epithelial detachment height $(\mu \mathrm{m})$ at the 12-month examination

\begin{tabular}{lcc}
\hline & Baseline & 12 Months \\
\hline All patients (33 eyes), median PED height (95\% CI) & $224(167-317)$ & $127(80-215, \mathrm{p}: 0.0001)$ \\
PRN regimen (14 eyes) & $262(188-362)$ & $174(113-342, \mathrm{p}: 0.01)$ \\
Fixed regimen group (19 eyes; loading phase+2q8) & $186(113-341)$ & 90 (0-177, p: 0.0002) \\
PRN group vs fixed regimen group & (p: 0.16) & (p: 0.07) \\
\hline
\end{tabular}

Abbreviations: fixed regimen, loading phase+bimonthly injections; PED, pigment epithelial detachment; PRN, pro re nata strategy.

Reduction in median PED Height was measured in both subgroups at the 12-month visit. The reduction reached statistical significance in both groups. 
No significant ocular or systemic adverse events were recorded over the course of the follow-up in both groups.

\section{Discussion}

We performed a retrospective study comparing the efficacy of two different treatment strategies (PRN vs fixed regimen) of aflibercept in nAMD patients with persistent fluid previously treated with ranibizumab. At 12 months of follow up, we observed an overall stabilization of visual acuity and an improvement in morphological findings in both treatment group.

In particular, the fixed regimen enabled the achievement of significant BCVA improvement, significant reduction in the percentage of patients with a vision loss of 5-15 ETDRS letters and a high percentage of patients with dry macula. It also reduced PED height to a greater extent than the PRN regimen at 12 months, confirming the efficacy of proactive treatment also in previously treated nAMD eyes.

The PRN regimen enabled the achievement of BCVA stabilization at 12 months associated with a reduction in retinal thickness, fluid and PED height at OCT, with a lower number of intravitreal injections (3.5), thus proving to be also a good choice in the management of switched eyes.

It has been well established that the efficacy of drugs blocking the increased level of intravitreal VEGF associated with nAMD may diminish over time. In addition to the mechanisms involving a change in the cellular composition of the neovascular membrane (pericyte coverage) and in the effects on the retinal architecture (fibrosis), the loss of activity has been partially attributed to tachyphylaxis or to increased tolerance. $^{22}$

At present the dilemma of when and how to change medications after their efficacy has diminished has not been addressed. ${ }^{23-26}$

Aflibercept is the last anti-VEGF drug that has been approved for the treatment of naive CNV associated with AMD, based on the efficacy demonstrated in the VIEW trials. ${ }^{8,9}$ In recent studies, it has been considered as a treatment option for patients presenting with persistent/ resistant nAMD or with high recurrence rate. ${ }^{9-20,23-26}$ Moreover, aflibercept has also been used as alternative treatment in patients treated successfully with ranibizumab or bevacizumab. ${ }^{19}$ Additional activity of aflibercept may rely on higher binding affinity to VEGF-A and the ability to target VEGF-B and PIGF. 5,6

Different treatment regimens of aflibercept have been adopted for switching therapy, but no clear recommendation has been made due to the lack of direct comparisons between treatment strategies.
For this reason, we aimed to compare the results obtained in two Italian retina centers after switching nAMD patients previously treated with anti-VEGF agents to aflibercept and treating them according to two different treatment strategies (PRN vs fixed regimen).

The analysis of the results by treatment regimen showed that BCVA remained stable in the PRN group for the whole follow-up period, while it improved significantly after the first three injections in the fixed regimen group and then stabilized up to month 12 . This resulted in a higher mean change in BCVA at 12 months in the fixed regimen group than in the PRN group (+2.5 vs -0.50 letters). Moreover, also the percentage of patients with BCVA loss of 5-15 letters was lower with the fixed regimen than with the PRN regimen (8 vs $25 \%$ ). The switch to aflibercept prevented severe loss of visual acuity (>15 letters): this was not observed in any patient in the fixed regimen group and in only one patient in the PRN regimen group.

Furthermore, the switch to aflibercept determined a significant reduction in CRT in both regimens. Although the absolute CRT reduction was higher in the fixed regimen group (148 vs 94 micron), the proportion of the thickness reduction was similar in the two groups $(-30.8 \%$ in fixed and $-27.2 \%$ in PRN regimen; $P=0.20)$. In addition, the proportion of patients with persisting IRF/ SRF was significantly reduced during the whole follow up in both groups: at month 12, the proportion of dry eyes was $58 \%$ in PRN group and $42 \%$ in fixed regimen group. Despite this difference, the proportion of dry patients between groups was not statistically significant at the end of follow up $(P=0.23)$.

At month 12, only four patients showed complete PED flattening, 3 in the PRN group (1 RAP lesion and 2 serous PED) and 1 in the fixed regimen group (RAP lesion). A greater reduction in the median subfoveal PED height was achieved at the 12-month visit in the fixed regimen (186 vs 90 micron, 52\% reduction) than in the PRN regimen (262 vs 174 micron, 31.9\% reduction). This could be related to the more prolonged VEGF blockade achieved in the fixed regimen.

Our long-term data on functional improvement in the fixed regimen confirm the results of previous studies with shorter follow-up, reporting on the efficacy of aflibercept administration in a course of at least three monthly injections in patients with persistent $\mathrm{CNV}$ activity. ${ }^{9,10,13}$

Kumar et $a l^{9}$ and Chang et $a l^{13}$ observed a statistically significant improvement by one line LogMAR and 6.9 letters at the 6-month examination, respectively. A comparison of their findings with our data is difficult due to differences in the baseline characteristics of the patients and study methodology.,13 
Also Singh et al ${ }^{19}$ demonstrated visual acuity improvement by 5.9 letters and a reduction in retinal thickness with the same fixed regimen at 6 months.

However, contrasting data on long-term outcome in terms of visual acuity have been reported. In the study of Messenger et al, ${ }^{15}$ where nAMD switched eyes have been treated at investigator discretion, and in the study of Grewal et al, ${ }^{16}$ where recalcitrant nAMD switched to aflibercept have been treated with a fixed regimen, a stabilization of visual acuity after 1 year, associated with anatomical improvement, was reported.

According to our study, other authors obtained a stabilization of visual acuity in the long-term follow up in chronic refractory and recurrent nAMD treated with aflibercept in PRN regimen. ${ }^{11,26}$

Overall, a statistically significant reduction in retinal thickness associated with a reduction in the proportion of eyes presenting IRF/SRF was reported in all published studies on nAMD patients switched to aflibercept. These positive outcomes were also confirmed in our study during the 12-month follow-up in both treatment regimen groups.

The long-term data of the current comparative study enables us to highlight different functional and anatomical outcomes with the two treatment regimens.

The results coming from the fixed regimen were obtained with seven intravitreal injections as per protocol. This result confirms that visual outcomes are strictly related to the number of injections. Nevertheless, the PRN regimen achieved results with a lower number of intravitreal injections (3.5), suggesting that it may also be a good choice for the management of switched eyes. In our PRN treated population $15.4 \%$ did not attend followup visits, and this lack of compliance could have influenced the visual outcomes in the long term.

Our study has some limitations. Indeed, the current study was not designed to compare the efficacy of the two treatment regimens in a randomized trial. It served as an exploratory analysis on the effects of a PRN regimen as an alternative to a fixed regimen in eyes with persistent $\mathrm{CNV}$ activity despite multiple ranibizumab injections. In addition, the involvement of two separate retina department, even if currently taking part to clinical trials, could introduce other biases. Furthermore, the relatively small sample prevented a subgroup analysis in an attempt to highlight different effects of aflibercept according to the CNV subtype.

In conclusion, the switch to aflibercept with both treatment strategies enabled improvement in morphological parameters and stabilization of visual function. Important reductions in CRT, fluid, and PED height associated with improvement in BCVA were achieved with the fixed regimen.

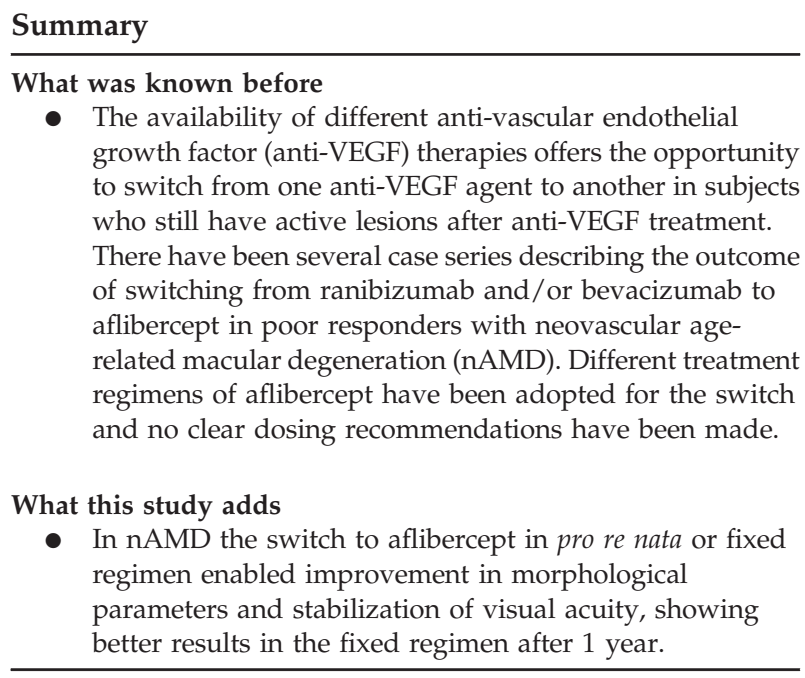

\section{Conflict of interest}

FR, MP, and MV reports personal fees from Allergan, personal fees from Bayer, personal fees from Novartis, outside the submitted work. The remaining authors declare no conflict of interest.

\section{Author contributions}

F Ricci and MP conceived and designed the study; F Ricci, $\mathrm{MP}, \mathrm{MV}$, and F Regine performed the analysis and interpretation; F Ricci, MP, MV, and FM wrote the article; F Ricci, MP, and MV carried out the critical revision of the article; F Ricci, MP, MV, F Regine, MT, and MS gave the final approval of the article; F Ricci, MP, MV, MS, and MT collected the data, F Ricci, MP, and MV provision of materials, patients, or resources, F Ricci, MP, and F Regine provided statistical expertise, MS, MP, and FM performed the Literature search. F Ricci and MP present the same rights to the article's intellectual properties.

\section{Acknowledgements}

The contribution of the IRCCS Fondazione Bietti in this paper was supported by the Italian Ministry of Health and by Fondazione Roma. We are thankful to Massimo Grossi and Susanna Lioi for data archiving.

\section{References}

1 Bressler NM. Age-related macular degeneration is the leading cause of blindness. JAMA 2004; 291: 1900-1901.

2 Brown DM, Kaiser PK, Michels M, Soubrane G, Heier JS, Kim RY et al. ANCHOR Study Group. Ranibizumab versus 
verteporfin for neovascular age-related macular degeneration. N Engl J Med 2006; 355: 1432-1444.

3 Rosenfeld PG, Brown DM, Heier JS, Boyer DS, Kaiser PK, Chung CY et al. MARINA Study Group. Ranibizumab for neovascular age-related macular degeneration. $N$ Engl J Med 2006; 355: 1419-1431.

4 Avery RL, Pieramici DJ, Rabena MD, Castellarin AA, Nasir MA, Giust MJ et al. Intravitreal bevacizumab (Avastin) for neovascular age-related macular degeneration. Ophthalmology 2006; 113: 363-372.

5 Papadopoulos N, Martin J, Ruan Q, Rafique A, Rosconi MP, Shi E et al. Binding and neutralization of vascular endothelial growth factor (VEGF) and related ligands by VEGF Trap, ranibizumab and bevacizumab. Angiogenesis 2012; 15: 171-185.

6 Rakic J, Lambert V, Devy L, Luttun A, Carmeliet P, Claes C et al. Placental growth factor, a member of the VEGF family, contributes to the development of choroidal neovascularization. Invest Ophthalmol Vis Sci 2003; 44: 3186-3193.

7 Heier JS, Brown DM, Chong V, Korobelnik JF, Kaiser PK, Nguyen QD et al. Intravitreal aflibercept (VEGF trap-eye) in wet age-related macular degeneration. Ophthalmology 2012; 119: $2537-2548$.

8 Schmidt-Erfurth U, Kaiser PK, Korobelnik JF, Brown DM, Chong V, Nguyen QD et al. Intravitreal aflibercept injection for neovascular age-related macular degeneration: ninetysix-week results of the VIEW studies. Ophthalmology 2014; 121: 193-201.

9 Kumar N, Marsiglia M, Mrejen S, Fung AT, Slakter J, Sorenson $\mathrm{J}$ et al. Visual and anatomical outcomes of intravitreal aflibercept in eyes with persistent subfoveal fluid despite previous treatments with ranibizumab in patients with neovascular age-related macular degeneration. Retina 2013; 33: 1605-1612.

10 Ho VY, Yeh S, Olsen TW, Bergstrom CS, Yan J, Cribbs BE et al. Short-term outcomes of aflibercept for neovascular agerelated macular degeneration in eyes previously treated with other vascular endothelial growth factor inhibitors. Am J Ophthalmol 2013; 156: 23-28.e2.

11 Yonekawa Y, Andreoli C, Miller JB, Loewenstein JI, Sobrin L, Eliott $\mathrm{D}$ et al. Conversion to aflibercept for chronic refractory or recurrent neovascular age-related macular degeneration. Am J Ophthalmol 2013; 156: 29-35.e2.

12 Bakall B, Folk JC, Boldt HC, Sohn EH, Stone EM, Russell SR et al. Aflibercept therapy for exudative age-related macular degeneration resistant to bevacizumab and ranibizumab. Am J Ophthalmol 2013; 156: 15-22.e1.

13 Chang AA, Li H, Broadhead GK, Hong T, Schlub TE, Wijeyakumar $\mathrm{W}$ et al. Intravitreal aflibercept for treatmentresistant neovascular age-related macular degeneration. Ophthalmology 2014; 121: 188-192.

14 Heussen FM, Shao Q, Ouyang Y, Joussen AM, Müller B. Clinical outcomes after switching treatment from intravitreal ranibizumab to aflibercept in neovascular age-related macular degeneration. Graefes Arch Clin Exp Ophthalmol 2014; 252: 909-915.

15 Messenger WB, Campbell JP, Faridi A, Shippey L, Bailey ST, Lauer AK et al. Injection frequency and anatomic outcomes 1 year following conversion to aflibercept in patients with neovascular age-related macular degeneration. $\mathrm{Br} \mathrm{J}$ Ophthalmol 2014; 98: 1205-1207.

16 Grewal DS, Gill MK, Sarezky D, Lyon AT, Mirza RG. Visual and anatomical outcomes following intravitreal aflibercept in eyes with recalcitrant neovascular age-related macular degeneration: 12-month results. Eye (Lond) 2014; 28: 895-899.

17 Gharbiya M, Iannetti L, Parisi F, De Vico U, Mungo ML, Marenco M. Visual and anatomical outcomes of intravitreal aflibercept for treatment-resistant neovascular age-related macular degeneration. Biomed Res Int 2014; 2014: 273754.

18 Arcinue CA, Ma F, Barteselli G, Sharpsten L, Gomez ML, Freeman WR. One-year outcomes of aflibercept in recurrent or persistent neovascular age-related macular degeneration. Am J Ophthalmol 2015; 159: 426-36.e2.

19 Singh RP, Srivastava S, Ehlers JP, Bedi R, Schachat AP, Kaiser PK. A single-arm, investigator-initiated study of the efficacy, safety and tolerability of intravitreal aflibercept injection in subjects with exudative age-related macular degeneration, previously treated with ranibizumab or bevacizumab: 6-month interim analysis. $\mathrm{Br} J$ Ophthalmol 2014; 98(Suppl 1): i22-i27.

20 Pinheiro-Costa J, Costa JM, Beato JN, Freitas-da-Costa P, Brandão E, Falcão MS et al. Switch to aflibercept in the treatment of neovascular AMD: one-year results in clinical practice. Ophthalmologica 2015; 233: 155-161.

21 Aiello L, Brucker A, Chang S, Cunningham Jr ET, D'Amico DJ, Flynn HW Jr et al. Evolving guidelines for intravitreous injections. Retina 2004; 24: S3-S19.

22 Binder S. Loss of reactivity in intravitreal anti-VEGF therapy: tachyphylaxis or tolerance? Br J Ophthalmol 2012; 96: $1-2$.

23 Ehlken C, Jungmann S, Böhringer D, Agostini HT, Junker B, Pielen A. Switch of anti-VEGF agents is an option for nonresponders in the treatment of AMD. Eye (Lond) 2014; 28: 538-545.

24 Wykoff CC, Brown DM, Maldonado ME, Croft DE. Aflibercept treatment for patients with exudative age-related macular degeneration who were incomplete responders to multiple ranibizumab injections (TURF trial). Br J Ophthalmol 2014; 98: 951-955.

25 He L, Silva RA, Moshfeghi DM, Blumenkranz MS, Leng T. Aflibercept for the treatment of retinal pigment epithelial detachments. Retina 2016; 36: 492-498.

26 Sarao V, Parravano M, Veritti D, Arias L, Varano M, Lanzetta P. Intravitreal Aflibercept for choroidal neovascularization due to age-related macular degeneration unresponsive to ranibizumab therapy. Retina 2016; 36: 770-777. 